\title{
Optimal state encoding for quantum walks and quantum communication over spin systems
}

\author{
Henry L. Haselgrove ${ }^{1,2, \text { : }}$ \\ ${ }^{1}$ School of Physical Sciences, University of Queensland, Brisbane 4072, Australia \\ ${ }^{2}$ Information Sciences Laboratory, Defence Science and Technology Organisation, Edinburgh 5111 Australia
}

(Dated: November 6, 2018)

\begin{abstract}
Recent work has shown that a simple chain of interacting spins can be used as a medium for high-fidelity quantum communication. We describe a scheme for quantum communication using a spin system that conserves $z$-spin, but otherwise is arbitrary. The sender and receiver are assumed to directly control several spins each, with the sender encoding the message state onto the larger state-space of her control spins. We show how to find the encoding that maximises the fidelity of communication, using a simple method based on the singular-value decomposition. Also, we show that this solution can be used to increase communication fidelity in a rather different circumstance: where no encoding of initial states is used, but where the sender and receiver control exactly two spins each and vary the interactions on those spins over time. The methods presented are computationally efficient, and numerical examples are given for systems having up to 300 spins.
\end{abstract}

\section{INTRODUCTION}

Quantum communication, the transfer of a quantum state from one place or object to another, is an important task in quantum information science 1]. The problem of communicating quantum information is profoundly different to the classical case 2, 3]. For example, quantum communication could not possibly be achieved by just measuring an unknown state in one place, and reconstructing in another. Rather, an entire system of source, target, and medium must evolve in a way that maintains quantum coherence.

In this paper we consider an idealised system of interacting spin- $1 / 2$ objects, isolated from the environment. The aim is to use the system's natural evolution to communicate a qubit state from one part of the system to another. The motivation is that such a system could be used as a simple "quantum wire" in future quantum information-processing devices. The most obvious configuration to choose is a simple one-dimensional open-ended chain, with interactions between nearest-neighbour spins, in which case we want the chain's evolution to transfer a qubit state from one end to the other. The methods in this paper apply to this simple type of chain, and also to spin networks of arbitrary graph.

A number of interesting proposals exist for quantum communication through spin chains. In [4], the 1D Heisenberg chain was considered, with coupling strengths constant over the length of the chain and with time. The idea was to initialise all spins in the "down" state, except the first spin, which was given the state of the qubit to be sent. After the system was allowed to evolve, the spin at the far end of the chain would then contain the sent state, to some level of fidelity. Simulations were carried out for a range of chain lengths, and it was shown that

*Electronic address: HLH@physics.uq.edu.au the fidelity was high only for very small chains.

In 5], a $1 \mathrm{D}$ spin chain with $X Y$ couplings was considered. Here, the coupling strengths were constant over time, but were made to vary over the length of the chain in a specific way. Like [4], the first spin was initialised in the state to be sent, with all other spins initialised to "down". It was shown that this scheme allows a perfect state transfer to the far spin site, for any length of chain.

In [6], a scheme was presented for high-fidelity quantum communication over a ring of spins with nearestneighbour Heisenberg couplings, using coupling strengths constant over the length of the ring and over time. The sender and receiver are located diametrically opposite to one another. The authors showed that excitations travel around the ring in a way that can be described using a concept from classical wave theory, the dispersion relation. Using this insight, they constructed a scheme where the sender, who controls several adjacent spins, constructs an initial state that is a Gaussian pulse having a particular group velocity chosen to minimise the broadening of the pulse over time. Using this state for the encoding of the $|1\rangle$ basis of the qubit message, and the all-down state as the encoding of $|0\rangle$, an arbitrary qubit can be sent with high fidelity over rings of any size, so long as the number of spins that the sender controls is at least the cube root of the total number.

Motivated by the results in [6], we pose the following problem. Say we are given the Hamiltonian for a system of interacting spins, where the graph of the interactions is not necessarily a ring structure, but is completely arbitrary. Also, the strength and type of interaction along each graph edge is arbitrary (so long as total $z$ spin is conserved). The sender Alice controls some given subset of the spins, and the receiver Bob controls some other given subset. How does Alice encode the qubit to be sent onto the spins she controls, in order to maximise the fidelity of communication? We know from [6] that the Gaussian pulse provides a near-optimal fidelity for the case of a Heisenberg ring (and is optimal in the limit of large ring 
sizes). What about other shapes of spin network? Can we find a general solution?

We provide a simple and efficient method for finding the maximum-fidelity encoding of the $|1\rangle$ message basis state, for a general $z$-spin-conserving spin system. (We assume that the encoding for the $|0\rangle$ basis state is fixed to the all-down state). So, unlike the schemes in [4], [5], and [6], which use systems with interactions that have specific strengths and conform to a specific graph, our scheme is designed to "make the most" of whatever arbitrary system is given to us. We give a numerical example of our method, for a system of 300 spins (where Alice and Bob each control 20 spins), showing a near-perfect average fidelity.

We give a second scheme for increasing fidelity, that does not use encoding of initial states, but relies on Alice and Bob dynamically controlling the interactions on their control spins. Here, the number of control spins is fixed at two each for Alice and Bob. We give a straightforward method for deriving control functions, that give a fidelity (and communication time) equal to the values that would result if Alice and Bob had instead each controlled many more spins (with static interactions) and used the optimal initial-state encoding scheme. This method has the combined benefits of being applicable to arbitrary $z$ spin-conserving spin-chains, yet having a fixed two-spin "interface" with Alice and Bob. We give numerical examples, and plot the derived control functions, for a 104-spin and a 29-spin system, showing a near-perfect fidelity in each case.

In the remainder of this introductory section, we briefly describe the assumptions behind our schemes, and define our notation. Sec. Idescribes our method of deriving the optimal message encoding. Sec. III describes our scheme for increasing fidelity via dynamic control. Concluding remarks are made in Sec. IV]

\section{A. Assumptions and notation}

The solution presented in this paper relies on two main assumptions, which we now list. Firstly, the system Hamiltonian must commute with $Z^{\text {tot }}$, which we define to be the $z$-component of the total spin operator

$$
\vec{\sigma}^{\mathrm{tot}} \equiv\left(X^{\mathrm{tot}}, Y^{\mathrm{tot}}, Z^{\mathrm{tot}}\right) \equiv \sum_{j} \vec{\sigma}_{j}
$$

where $\vec{\sigma}_{j}$ is the vector of Pauli operators $\left(\sigma^{x}, \sigma^{y}, \sigma^{z}\right)$ acting on the $j$-th spin. The Pauli operators in the basis "down" $|\downarrow\rangle$ and "up" $|\uparrow\rangle$ are

$$
\sigma^{x}=\left[\begin{array}{ll}
0 & 1 \\
1 & 0
\end{array}\right] ; \quad \sigma^{y}=\left[\begin{array}{rr}
0 & -i \\
i & 0
\end{array}\right] ; \quad \sigma^{z}=\left[\begin{array}{rr}
1 & 0 \\
0 & -1
\end{array}\right] .
$$

Secondly, the spin system must be initialised to the alldown state $|\downarrow\rangle \otimes \cdots \otimes|\downarrow\rangle$, before the communication is carried out. Note that the schemes in [4], $[5]$ and $[\underline{6}]$ also make use of these two assumptions. It could be argued that the first condition is reasonable because it follows from rotational invariance. Of course, any external magnetic field will destroy this invariance, and in particular any magnetic field which is not in the $z$ direction will mean that the $z$-component of total spin is no longer conserved. The Heisenberg and $X Y$ interactions are examples of interactions that conserve $Z^{\text {tot }}$. The second constraint might be rather difficult to achieve in practice. One possibility would be to apply a strong polarising magnetic field in the $z$ direction, over the entire system, and let the system relax to its ground state.

In the remainder of the paper, in place of the notation $|\downarrow\rangle$ and $|\uparrow\rangle$ for the eigenstates of $\sigma^{z}$, we will use the equivalent but more convenient notation $|0\rangle$ and $|1\rangle$. A computational basis state of the system is defined to be one where each spin is in either a $|0\rangle$ state or a $|1\rangle$ state. Note that the computational basis states are all eigenstates of $Z^{\text {tot }}$, and the eigenvalue has one of $N+1$ possible values, given by the number of $|0\rangle \mathrm{s}$ minus the number of $|1\rangle$ s. So in a system of $N$ spin- $1 / 2$ objects, we can break the state space into $N+1$ subspaces of different well-defined $z$-component of total spin. We use $\mathcal{H}^{(n)}, n=0, \ldots, N$, to denote these subspaces. $\mathcal{H}^{(n)}$ is the eigenspace of $Z^{\text {tot }}$ that is spanned by the $\left(\begin{array}{l}N \\ n\end{array}\right)$ computational basis states that have $n$ qubits in the $|1\rangle$ state and the rest in the $|0\rangle$ state.

Since the system Hamiltonian $H$ commutes with $Z^{\text {tot }}$, a state in $\mathcal{H}^{(n)}$ will remain in $\mathcal{H}^{(n)}$ under the evolution of $H$. $\mathcal{H}^{(0)}$ is one-dimensional; it is spanned by the all-zero state $|0\rangle \otimes \ldots \otimes|0\rangle$. So this state is a stationary state of $H$.

\section{THE OPTIMAL ENCODING SCHEME}

Say that Alice wishes to send the qubit state $\alpha|0\rangle+$ $\beta|1\rangle$. In our scheme, she does so by preparing the state $\alpha|\mathbf{0}\rangle_{A}+\beta\left|1_{E N C}\right\rangle_{A}$, where $|\mathbf{0}\rangle_{A}$ is the all-zero state on her spins, and $\left|1_{E N C}\right\rangle_{A}$ is some state orthogonal to $|\mathbf{0}\rangle_{A}$ (the " $E N C$ " stands for "encoded"). (Note that Alice doesn't necessarily know $\alpha$ and $\beta$. She would presumably prepare the state by some unitary operation acting on her spins and some external spin containing the state $\alpha|0\rangle+\beta|1\rangle$.) We assume that the entire spin chain is initialised to the all-zero state, so immediately after Alice prepares the abovementioned state on her spins, the state of the whole system is

$$
|\Psi(0)\rangle \equiv\left(\alpha|\mathbf{0}\rangle_{A}+\beta\left|1_{E N C}\right\rangle_{A}\right) \otimes|\mathbf{0}\rangle_{\bar{A}}
$$

where $\bar{A}$ refers to all spins that Alice does not control. The whole spin system is allowed to evolve for a time $T$, giving the state $|\Psi(T)\rangle=e^{-i H T}|\Psi(0)\rangle$. Using the fact that $|0\rangle \otimes \cdots \otimes|0\rangle$ is a stationary state, $|\Psi(T)\rangle$ can be written (up to some global phase) as

$$
\begin{aligned}
&|\Psi(T)\rangle=\beta \sqrt{1-\mathcal{C}_{B}(T)}|\eta(T)\rangle+ \\
&|\mathbf{0}\rangle_{\bar{B}}\left(\alpha|\mathbf{0}\rangle_{B}+\beta \sqrt{\mathcal{C}_{B}(T)}|\gamma(T)\rangle_{B}\right),
\end{aligned}
$$


for some nonnegative $\mathcal{C}_{B}(T)$, some normalised $|\gamma(T)\rangle_{B}$ orthogonal to $|\mathbf{0}\rangle_{B}$, and for some normalised $|\eta(T)\rangle$ that is orthogonal to all states of the form $|0\rangle_{\bar{B}} \otimes|v\rangle_{B}$.

We now show that $\mathcal{C}_{B}(T)$ can be used as a measure of success. Comparing Eqs. (3) and (44), we see that $\mathcal{C}_{B}(0)=0$. If $\mathcal{C}_{B}(T)$ reaches 1 for some later $T$, a perfectfidelity quantum communication has resulted. This is because Bob will then have the state $\alpha|\mathbf{0}\rangle_{B}+\beta|\gamma\rangle_{B}$ on the qubits he controls, which can be "decoded" by a unitary operation into the state $\alpha|0\rangle+\beta|1\rangle$ of a single spin, since $|\mathbf{0}\rangle_{B}$ and $|\gamma\rangle_{B}$ are orthogonal. If $\mathcal{C}_{B}(T)$ is less than 1, the unitary decoding by Bob will leave him with a qubit state $\rho$ that is generally different to the message state. That is, the measure of state fidelity $F \equiv(\alpha|0\rangle+\beta|1\rangle)^{\dagger} \rho(\alpha|0\rangle+\beta|1\rangle)$ between the message $\alpha|0\rangle+\beta|1\rangle$ and $\rho$, will generally be less than one whenever $\mathcal{C}_{B}(T)<1$. However, the value of $F$ is highly dependent on the message state - for example, if $\alpha=1$ then $F=1$ regardless of the value of $\mathcal{C}_{B}(T)$.

$\mathcal{C}_{B}(T)$, on the other hand, is a message-independent measure of the fidelity of communication. Consider $\bar{F}$, defined to be the state fidelity $F$ averaged over all message states. For encodings $\left|1_{E N C}\right\rangle$ that belong to the $\mathcal{H}^{(1)}$ subspace, we have

$$
\bar{F}=\frac{1}{2}+\frac{1}{3} \sqrt{\mathcal{C}_{B}(T)}+\frac{1}{6} \mathcal{C}_{B}(T),
$$

which is a monotonic function of $\mathcal{C}_{B}(T)[\underline{6}$. So, in this case maximising the average state fidelity is equivalent to maximising $\mathcal{C}_{B}(T)$. More generally, for $\left|1_{E N C}\right\rangle$ not in $\mathcal{H}^{(1)}$, the expression in Eq. (5) provides a reasonably tight lower bound on $\bar{F}$ :

$$
\begin{aligned}
\frac{1}{2}+\frac{1}{3} \sqrt{\mathcal{C}_{B}(T)}+\frac{1}{6} \mathcal{C}_{B}(T) \leq \bar{F} & \leq \frac{1}{2}+\frac{1}{3} \sqrt{\mathcal{C}_{B}(T)}+\frac{1}{6} \\
& =\frac{2}{3}+\frac{1}{3} \sqrt{\mathcal{C}_{B}(T)} .
\end{aligned}
$$

The precise value of $\bar{F}$ will then depend on $|\eta(T)\rangle$ and the full specification of Bob's decoding unitary.

A further argument for using $\mathcal{C}_{B}(T)$ as a measure of communication fidelity comes from considering the system's ability to transfer quantum entanglement from Alice to Bob. Suppose that Alice, instead of sending a message which is a pure quantum state $\alpha|0\rangle+\beta|1\rangle$, sends a state which is maximally entangled with some additional spin that Alice possesses. (The additional spin does not interact when the system evolves). If the communication is perfect, the result must be that Bob's decoded message becomes maximally entangled with Alice's additional spin. So more generally, when the communication is not perfect, the amount of entanglement generated between Alice and Bob would be a good measure of communication fidelity. In fact, the entanglement generated, measured by the concurrence, is equal to $\sqrt{\mathcal{C}_{B}(T)}$ (a proof of this fact is outlined in Appendix A). This is independent of $|\eta(T)\rangle$, or the full specification of Bob's decoding unitary, or whether $|1\rangle_{E N C}$ belongs to $\mathcal{H}^{(1)}$.
To recap, when the Hamiltonian commutes with $Z^{\text {tot }}$ and the state is initialized to $|\mathbf{0}\rangle$, the problem of achieving a high communication fidelity can be boiled down to choosing an appropriate initial encoding $\left|1_{E N C}\right\rangle$ for the $|1\rangle$ qubit basis state. We seek a state $\left|1_{E N C}\right\rangle_{A} \otimes|\mathbf{0}\rangle_{\bar{A}}$ that has the property that it evolves to (or near to) a state of the form $|\mathbf{0}\rangle_{\bar{B}} \otimes|\gamma\rangle_{B}$, or in other words such that $\mathcal{C}_{B}(T) \approx 1$ for some $T$. Alice's choice for the "encoding" of the $|0\rangle$ qubit basis state is fixed to the all-zero state. With perfect fidelity that basis state will evolve to the all-zero state on Bob's spins. (Note that in some cases it may be possible to increase fidelity further by allowing an encoding for $|0\rangle$ other than the all-zero state. We ignore such a possibility, in order to keep the method for finding the encoding simple and efficient. The simplification is used likewise in [7].)

We now show that the encoding $\left|1_{E N C}\right\rangle$ which maximises $\mathcal{C}_{B}(T)$ for a given $T$ can be found by performing the singular value decomposition on a modified version of the evolution matrix $e^{-i H T}$.

Let $\mathcal{A}$ be the vector subspace of states of the form $\left|1_{E N C}\right\rangle_{A} \otimes|\mathbf{0}\rangle_{\bar{A}}$, such that ${ }_{A}\left\langle\mathbf{0} \mid 1_{E N C}\right\rangle_{A}=0$. Similarly, let $\mathcal{B}$ be the vector subspace of states of the form $|\mathbf{0}\rangle_{\bar{B}} \otimes$ $|\gamma\rangle_{B}$, such that ${ }_{B}\langle\mathbf{0} \mid \gamma\rangle_{B}=0$. In other words, $\mathcal{A}$ reflects all the possible encodings that Alice could use for the $|1\rangle$ qubit basis state (regardless of the fidelity they would achieve). $\mathcal{B}$ is the set of states that we would like some state in $\mathcal{A}$ to evolve to; a state in $\mathcal{A}$ that evolves to one in $\mathcal{B}$ represents an encoding for $|1\rangle$ that gives $\mathcal{C}_{B}(T)=1$ and thus a perfect average fidelity.

Let $P_{\mathcal{A}}$ and $P_{\mathcal{B}}$ be the projectors onto the subspaces $\mathcal{A}$ and $\mathcal{B}$. Let $U(T) \equiv e^{-i H T}$ be the time-evolution operator. From Eqs. (3) and (4), we can write

$$
\mathcal{C}_{B}(T)=\| P_{\mathcal{B}} U(T)\left|1_{E N C}\right\rangle_{A} \otimes|\mathbf{0}\rangle_{\bar{A}} \|^{2},
$$

where $\|\cdot\|$ denotes the $l_{2}$-norm. This means that for a particular total communication time $T$, choosing the optimal initial encoding for the $|1\rangle$ state is a matter of finding the normalised $|\psi\rangle \in \mathbb{C}^{2^{N}}$ that maximises $\| P_{\mathcal{B}} U(T) P_{\mathcal{A}}|\psi\rangle \|$. The maximum value is given by the largest singular value of $\tilde{U}(T) \equiv P_{\mathcal{B}} U(T) P_{\mathcal{A}}$, and the corresponding optimal $|\psi\rangle$ is the first right-singular-vector of $\tilde{U}(T)[]]$. Recall, the SVD (singular value decomposition) of $\tilde{U}(T)$ is

$$
\begin{aligned}
& \tilde{U}(T)=V S W^{\dagger}
\end{aligned}
$$

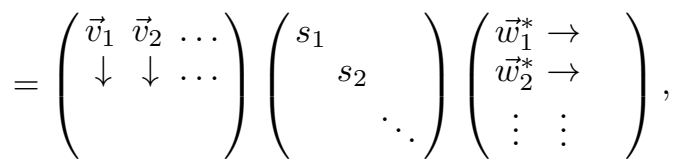

where $s_{1} \geq s_{2} \geq \cdots \geq 0$ are the singular values, the orthonormal $\vec{w}_{j}$ are the right singular vectors, and the orthonormal $\vec{v}_{j}$ are the left singular vectors of $\tilde{U}(T)$. Numerical packages such as Matlab have in-built routines for easily calculating the SVD. So, we have that $\mathcal{C}_{B}(T)$ has its maximum value, $s_{1}^{2}$, when Alice chooses the initial state $\left|1_{E N C}\right\rangle_{A} \otimes|\mathbf{0}\rangle_{\bar{A}}=\vec{w}_{1}$ to encode $|1\rangle$. 
Other parts of the decomposition could be useful as well. Say Alice wants to transmit two qubits simultaneously to Bob. If she uses the all-down state to encode the $|00\rangle$ basis state, then she should use the encodings $\vec{w}_{1}$, $\vec{w}_{2}$, and $\vec{w}_{3}$ for the other three basis states $|01\rangle,|10\rangle$, and $|11\rangle$. Then, so long as $s_{3} \approx 1$, the two qubits would be simultaneously communicated with high fidelity.

The vectors $\vec{w}_{j}$ and the values $s_{j}$ are also the eigenvectors and square-root eigenvalues respectively of $\left(P_{B} \tilde{U}(T) P_{A}\right)^{\dagger} P_{B} \tilde{U}(T) P_{A}=P_{A} \tilde{U}^{\dagger}(T) P_{B} \tilde{U}(T) P_{A}$. Now, $Z^{\text {tot }}$ commutes with $P_{A} \tilde{U}^{\dagger}(T) P_{B} \tilde{U}(T) P_{A}$ because it commutes with each of $P_{A}, P_{B}, \tilde{U}(T)$, and $\tilde{U}^{\dagger}(T)$ separately. So the $\vec{w}_{j}$ will all have well-defined total $Z$ spin (or can be chosen to, wherever ambiguities exist because of degeneracies in the $s_{j}$ ). This is important when it comes to calculating these solutions efficiently. Instead of performing the full $2^{N}$ by $2^{N}$ matrix exponential and SVD, the calculation can be done separately for each of the smaller subspaces $\mathcal{H}^{(n)}$, starting each calculation with the $\left(\begin{array}{l}N \\ n\end{array}\right)$ by- $\left(\begin{array}{l}N \\ n\end{array}\right)$ part of the Hamiltonian that acts on the $\mathcal{H}^{(n)}$ subspace.

Alice can't create a state with more than $|A|$ qubits in the "one" state, where $|A|$ is the number of spins she controls. So, in fact the calculation only needs to be done over the $\mathcal{H}^{(1)}, \ldots, \mathcal{H}^{(|A|)}$ subspaces (in other words, the singular values corresponding to states in other subspaces will always be zero).

In practice we have found that the optimal solution $\vec{w}_{1}$ often belongs to the $\mathcal{H}^{(1)}$ subspace. (In particular, we calculated the optimal solution for a range of different values of $T$ for various 8 and 9 -spin systems, and found that only for a very small minority of the values of $T$, for each system, was the solution not in the $\mathcal{H}^{(1)}$ subspace ). A rudimentary argument for this can be made as follows. Looking for solutions in $\mathcal{H}^{(n)}$ means optimising over Alice's $\left(\begin{array}{c}|A| \\ n\end{array}\right)$ degrees of freedom (of the space $\mathcal{A} \cap \mathcal{H}^{(n)}$ ), in order to make the final state land in or near a $\left(\begin{array}{c}|B| \\ n\end{array}\right)$ dimensional target space $\mathcal{B} \cap \mathcal{H}^{(n)}$. This must be achieved despite the fact that the Hamiltonian is "trying" to move the state through a much larger $\left(\begin{array}{l}N \\ n\end{array}\right)$-dimensional space $\mathcal{H}^{(n)}$. Over the various values of $n=1, \ldots,|A|$, the dimensionality of $\mathcal{A} \cap \mathcal{H}^{(n)}$ and $\mathcal{B} \cap \mathcal{H}^{(n)}$ as a fraction of the dimensionality of $\mathcal{H}^{(n)}$ is largest when $n=1$. In other words, when $n=1$, the size of the target space, and amount of control available of the initial state, is largest as a fraction of the dimensionality of the entire subspace $\mathcal{H}^{(n)}$

So, in general we can restrict all the calculations to the $N$-dimensional subspace $\mathcal{H}^{(1)}$, and there will still be a good chance that we will arrive exactly at the globallyoptimal encoding $\vec{w}_{1}$. Ignoring solutions in the other subspaces will increase the efficiency of calculation considerably, especially for large chains.

The evolution of a state in the $\mathcal{H}^{(1)}$ subspace can also be interpreted as a continuous quantum walk of a particle over a graph. (For an introduction to quantum walks, see for example [9] and references therein). The

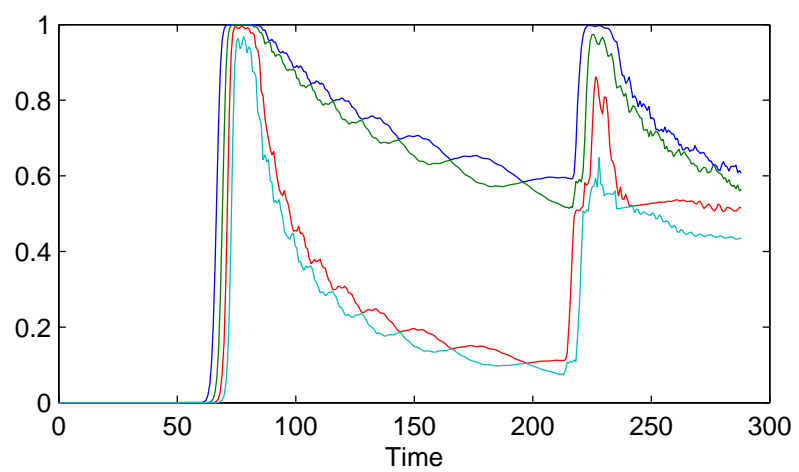

FIG. 1: The largest four singular values of $\tilde{U}^{(1)}(T)$, for a range of communications times $T$. Open-ended Heisenberg chain, $N=300$, and Alice and Bob each control 20 sites.

graph is simply the graph of interactions between spins in the Hamiltonian $H$, and the state $|\mathbf{0}\rangle_{\bar{j}} \otimes|1\rangle_{j}$ corresponds to the particle being at vertex $j$ of that graph. So our methods for increasing communication fidelity are, equivalently, methods for guiding a quantum walk from one part of a graph to another. We point out this connection because of the significant interest currently in using quantum walks for solving computational problems (see for example [7, 10, 11] and references therein).

To demonstrate the use of the SVD optimal-encoding technique, we now consider a numerical example. Imagine that Alice and Bob are joined by a 300-site openended chain with nearest-neighbour couplings given by the antiferromagnetic Heisenberg interaction, with coupling strengths all equal to 1 . That is,

$$
H=\sum_{j=1}^{299} \vec{\sigma}_{j} \cdot \vec{\sigma}_{j+1} .
$$

Assume that Alice and Bob control the first and last 20 spins respectively.

In light of the earlier discussion, we restrict our optimisation to the $\mathcal{H}^{(1)}$ subspace, and thus ignore all singular vectors in other subspaces. A Matlab program is used to carry out the following calculations. First, the 300 by 300 matrix $H^{(1)}$, defined to be the part of $H$ that acts on $\mathcal{H}^{(1)}$, is constructed. Then, the SVD of

$$
\tilde{U}^{(1)}(T)=P_{\mathcal{B} \cap \mathcal{H}^{(1)}} e^{-i H^{(1)} T} P_{\mathcal{A} \cap \mathcal{H}^{(1)}}
$$

is calculated for a range of values of $T$. The optimal value for communication time is not known beforehand, so this repetition of the calculation for different values of $T$ is needed in order to find a reasonable tradeoff between communication time and fidelity.

The four largest singular values, $s_{1}, \ldots, s_{4}$, of $\tilde{U}^{(1)}(T)$ are plotted in Figure! Over the range of $T$ shown, $s_{1}(T)$ has its maximum of 0.99999 at $T=75.75$. So, this system can transmit a qubit with near-perfect fidelity, over 


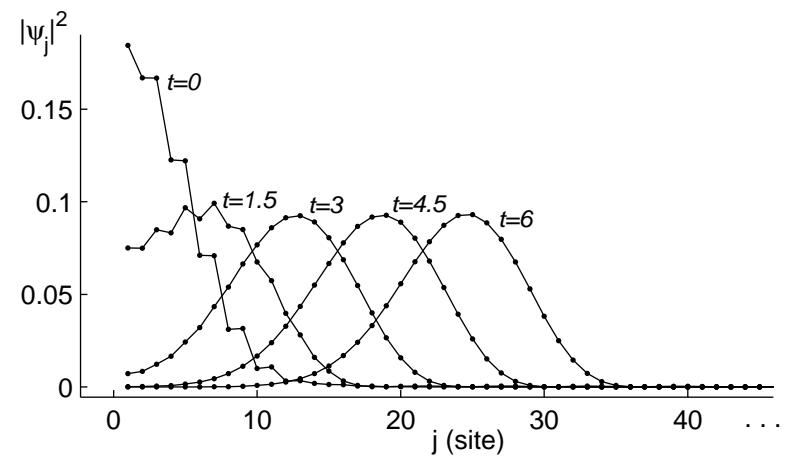

FIG. 2: The optimal encoded state $\vec{w}_{1}(75.75)$, evolved for a sequence of times $t$.

a time interval of 75.75 . The graph shows that $s_{2}(75.75)$ and $s_{3}(75.75)$ are also very close to 1 , so in fact two qubits could be transmitted simultaneously with high fidelity in this example, using the encodings $|\mathbf{0}\rangle, \vec{w}_{1}(75.75)$, $\vec{w}_{2}(75.75)$ and $\vec{w}_{3}(75.75)$ for the two-qubit basis states.

Let's look at the actual optimally encoded states that are generated in this example. We visualise a state in $\mathcal{H}^{(1)}$ by plotting the square magnitude of the coefficients $\psi_{j}$, where $\psi_{j}$ is the coefficient of the basis state that has the $j$-th spin in the $|1\rangle$ state:

$$
\mathcal{H}^{(1)} \ni|\psi\rangle=\sum_{j=1, \ldots, N} \psi_{j}|1\rangle_{j} \otimes|\mathbf{0}\rangle_{\bar{j}} .
$$

In Figure 22 we show the evolution of the state $\vec{w}_{1}(75.75)$. That is, we set $|\psi(0)\rangle=\vec{w}_{1}(75.75)$, and $|\psi(t)\rangle=e^{-i H^{(1)}}|\psi(0)\rangle$, and plot the magnitudes $\left|\psi_{j}\right|^{2}$ for a sequence of equally-spaced times $t$. As is necessarily the case, the $t=0$ state has non-zero coefficients only on Alice's spins, $j=1 \ldots 20$. The state deforms itself into a Gaussian shape quite quickly. This is interesting in comparison with the results in [6]. Whilst Gaussian initial states were shown to optimise fidelity on a Heisenberg ring, the best initial states for an open-ended Heisenberg chain are ones that deform into a Gaussians. From the total communication time in this example, the group velocity of the pulse is roughly 3.95 (defining the distance between neighbouring spins to be 1 ). Thus, in the open-ended Heisenberg chain we have found the same phenomena that appeared in the Heisenberg ring in [6], notably that the system has a preferred group velocity that gives a minimum dispersion and thus maximum fidelity. This explains the fact that in Figure 1 the singular values drop for $T$ greater than 75.75 , and rise again to a near-maximum at $T \approx 225 \approx 3 \times 75.75$ : the high-fidelity communication for $T \approx 225$ is also operating at the preferred group velocity, but the wave packet is traversing the chain three times, after bouncing from each end.

Curiously, the lower solutions $\vec{w}_{2}(75.75)$ and $\vec{w}_{3}(75.75)$ seem to evolve into a sum of two and three Gaussians re-

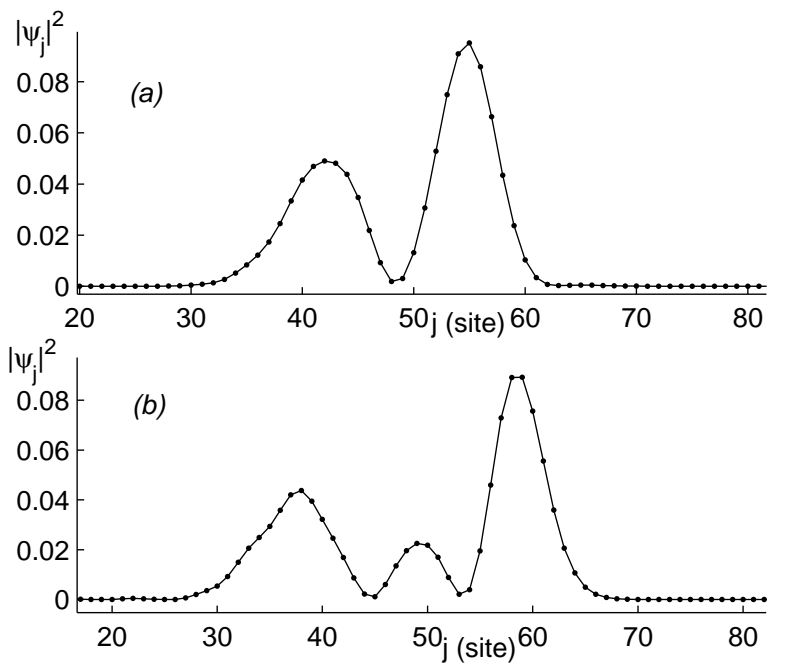

FIG. 3: The states $(a) \vec{w}_{2}(75.75)$ and $(b) \vec{w}_{3}(75.75)$ evolved for time $=12$.

spectively (see Figure 3). Animations of these evolutions are available online [12].

\section{DYNAMIC CONTROL}

The scheme we presented in Sec. III utilises the evolution of a system having completely static interactions. The control that Alice and Bob have over the chain is only for an instant at the beginning and end of the procedure, and so their only degrees of freedom for increasing the fidelity lie in the encoding they use. For very long chains, the number of control sites needed to give a high fidelity might become impractically large, as suggested by the results in [6]. In this section, we consider the advantage that can be gained by allowing Alice and Bob to control their spins throughout the procedure, by modulating the strength of interactions on those spins. The advantages of the scheme are that the number of control spins are fixed at four, and that suitable functions for Alice and Bob to use to vary the interaction strengths are easily derived from a simple extension of the SVD approach already described.

This type of control scheme is an example of a fundamental problem in quantum information processing, that of determining how to use the limited physical control that one has of a quantum system, in such a way as to achieve the dynamics that are required. For the task at hand, our method provides a practical and efficient way of finding an appropriate dynamical control.

A general schematic for the system is shown in Figure 4. Alice is now in control of just two spins, labeled $A_{1}$ and $A_{2}$, and, likewise, Bob controls two spins $B_{1}$ and $B_{2}$. All the other spins in the system are collectively denoted $C$. The graph of the interactions connecting Alice and 


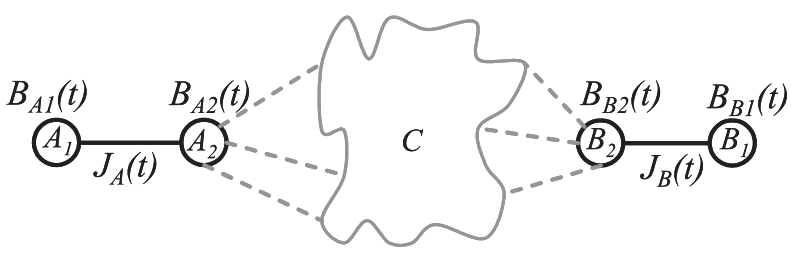

FIG. 4: The general setup, whereby Alice and Bob modulate a total of six parameters of the Hamiltonian in order to increase communication fidelity.

Bob's spins can be arbitrary, except that $A_{1}$ must directly couple only to $A_{2}$, and $B_{1}$ must directly couple only to $B_{2}$. Like the previous scheme, we require that the system Hamiltonian $H$ commutes with $Z^{\text {tot }}$, and that all spins are initialised to $|0\rangle$ before the procedure starts. So again the problem is that of finding a way of sending the $|1\rangle$ basis state with high fidelity.

The protocol works as follows. At time $t=0, \mathrm{Al}-$ ice transfers the qubit state she wishes to send onto the spin $A_{1}$. Then she varies the coupling strength between $A_{1}$ and $A_{2}$ according to some function which we denote $J_{A}(t)$, and varies the $z$ magnetic field on $A_{1}$ and $A_{2}$ according to functions $B_{A 1}(t)$ and $B_{A 2}(t)$. At the same time, Bob varies his coupling strength and $z$ magnetic fields according to the functions $J_{B}(t), B_{B 1}(t)$ and $B_{B 2}(t)$. This process is continued over some time interval $0<t<T$. At $t=T$ Bob's spin $B_{1}$ will contain the sent qubit state, to a level of fidelity that depends on the six control functions.

How do we choose the control functions in a way that gives us a high average communication fidelity? The trick is to imagine a modified version of the system, where a number of "phantom" spins have been added to both Alice and Bob's set of control spins, but all couplings are now fixed (see Figure 5).

The SVD method is applied to this modified system, to find the optimal initial state on Alice's extended set of control spins. The evolution of the encoded state through the modified system is then simulated on a (classical) computer, and the results of the simulation are used to derive appropriate control functions for the actual physical system, using a method which we describe below. Since, over the bulk of the physical system, the initial state and interactions are identical to those at the corresponding regions of the modified system, the problem reduces to finding control functions which make the state on $A_{2}$ and $B_{2}$ in the physical system evolve in the same way as those corresponding spins in the modified chain. When that is achieved, the state on the bulk of the physical chain will evolve in the same way as in the modified chain, which means that we can communicate a qubit with the same fidelity as for the optimally encoded state in the modified system.

For the sake of clarity, we describe the method in detail for a less general configuration, the 1D $X Y$ chain. The

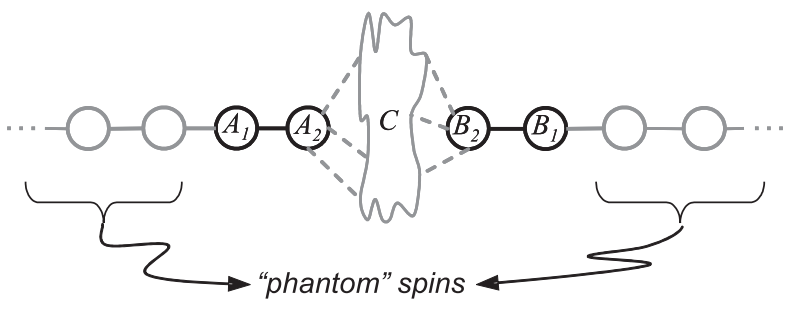

FIG. 5: This is a modified version of Figure (4). Calculating the optimal initial encoded state on this system will help Alice and Bob derive suitable control functions for their actual physical setup in Figure (4).

derivation is simpler in this case because, as we shall see, the magnetic control is not needed. The system Hamiltonian is given by

$$
\begin{aligned}
H(t)=\sum_{j=2}^{N-2} & J_{j}\left[\sigma_{j}^{x} \sigma_{j+1}^{x}+\sigma_{j}^{y} \sigma_{j+1}^{y}\right] \\
& \quad+J_{A}(t)\left(\sigma_{1}^{x} \sigma_{2}^{x}+\sigma_{1}^{y} \sigma_{2}^{y}\right) \\
& \quad+J_{B}(t)\left(\sigma_{N-1}^{x} \sigma_{N}^{x}+\sigma_{N-1}^{y} \sigma_{N}^{y}\right) .
\end{aligned}
$$

That is, there are some arbitrary fixed $J_{j}$ that specify the strengths of the $X Y$ couplings over the bulk of the chain. The strengths at the first and last links can be varied over time by Alice and Bob.

We write down a Hamiltonian $\tilde{H}$ of a modified system, where we have extended the length of the chain in both directions by adding $N_{P}$ phantom spins to both Alice and Bob's sides. The new coupling strengths are chosen to be 1 , and the two strengths that were time-varying in the original system are now also fixed at one. So that we can use the same numbering system for the spins as in Eq. (12), we let the indices of the spins range into the negative in the modified system, running from $1-N_{P}$ to $N+N_{P}$. The modified Hamiltonian is written simply as

$$
\tilde{H}=\sum_{j=1-N_{P}}^{N+N_{P}-1} J_{j}\left[\sigma_{j}^{x} \sigma_{j+1}^{x}+\sigma_{j}^{y} \sigma_{j+1}^{y}\right],
$$

where we have extended the definition of $J_{j}$ so that it equals 1 for $j=\left(1-N_{P}\right), \ldots, 1$ and for $j=(N-$ $1), \ldots,\left(N+N_{P}-1\right)$.

Next, using the SVD method, we find the best encoded initial state on the set of spins from index $\left(1-N_{P}\right)$ to 1 , while assuming that the target set of spins ranges from $N$ to $\left(N+N_{P}-1\right)$. That is, we are imagining a "modified Alice" that controls the first $\left(N_{P}+1\right)$ spins and a "modified Bob" that controls the last $\left(N_{P}+1\right)$ spins of this extended chain. Recall that the SVD method depends on a choice of total communication time $T$. As in the example earlier, we may wish to search over a range of values of $T$ to find the most suitable value. It is important for the procedure at hand that we restrict ourselves to states 
in the $\mathcal{H}^{(1)}$ subspace (whereas before this restriction was just a way of making the solution much faster to compute). So, we calculate $\vec{w}_{1}(T)$, the first right-singularvector of $P_{\mathcal{B} \cap \mathcal{H}^{(1)}} e^{-i \tilde{H}^{(1)} T} P_{\mathcal{A} \cap \mathcal{H}^{(1)}}$, where $\tilde{H}^{(1)}$ is the part of $\tilde{H}$ that acts on the $\mathcal{H}^{(1)}$ subspace.

Then, we need to be able to calculate the evolution of the state $\vec{w}_{1}$, over a range of times $t$ from 0 to $T$. Let $|\psi(0)\rangle=\vec{w}_{1}(T)$, and $|\psi(t)\rangle=e^{-i H^{(1)}}|\psi(0)\rangle$. As earlier, the evolving state is a series of complex coefficients $\psi_{j}(t)$, where $j$ is the index to a spin site, ranging from $\left(1-N_{P}\right)$ to $\left(N+N_{P}-1\right)$. We need to know $\psi_{1}(t)$ and $\psi_{N}(t)$ for every value of $t$ that we wish to calculate $J_{A}(t)$ and $J_{B}(t)$ for.

Similarly we use $\phi_{j}(t), j=1, \ldots, N$ to denote the evolution of the $|1\rangle$ qubit state over the original physical chain. Recall that in this scheme, Alice places the qubit state to be sent, unencoded, onto spin number 1, after all other spins have been initialised to zero. So, initially we have $\phi_{j}(0)=\delta_{j, 1}$. The functions $\phi_{j}(t)$ depend on the control functions $J_{A}(t)$ and $J_{B}(t)$ (whereas the $\psi_{j}(t)$ do not).

The aim is to chose control functions $J_{A}(t)$ and $J_{B}(t)$ in such a way as to force $\phi_{j}(t)=\psi_{j}(t)$, for all the spins in the range $j=2, \ldots, N-1$, and for all $t$ in the interval $[0, T]$. That is, we know the way the optimal encoded state evolves over the modified chain, and we want to make the $|1\rangle$ state in the physical system evolve in exactly the same way, over all spins except 1 and $N$. In this way, the physical system will carry a qubit across it's length with the same fidelity as the encoded modified system does.

The interactions on the spins from site 3 to site $N-2$ are the same in the physical chain as in the modified chain. So, the differential equations for the $\psi_{j}(t)$ are the same as those for the $\phi_{j}(t)$, for $j=3, \ldots, N-2$. Specifically,

$$
\begin{aligned}
& \frac{d \psi_{j}(t)}{d t}=-2 i\left[J_{j-1} \psi_{j-1}(t)+J_{j} \psi_{j+1}(t)\right] \quad \text { and } \\
& \frac{d \phi_{j}(t)}{d t}=-2 i\left[J_{j-1} \phi_{j-1}(t)+J_{j} \phi_{j+1}(t)\right],
\end{aligned}
$$

for $j=3, \ldots, N-2$. Also, the initial conditions are the same between the $\psi_{j}$ and the $\phi_{j}$, for $j=2, \ldots,(N-1)$ : $\psi_{j}(0)=\phi_{j}(0)=0$.

It follows that if we can use our control functions to force $\frac{d \phi_{2}(t)}{d t}=\frac{d \psi_{2}(t)}{d t}$, and $\frac{d \psi_{N-1}(t)}{d t}=\frac{d \phi_{N-1}(t)}{d t}$, over the time range $t=0, \ldots, T$, then we will have $\psi_{j}(t)=\phi_{j}(t)$ for all $t$ in that time range, and for all $j=2, \ldots, N-1$, as desired.

Now,

$$
\frac{d \psi_{2}(t)}{d t}=-2 i\left[\psi_{1}(t)+J_{2} \psi_{3}(t)\right]
$$

and

$$
\frac{d \phi_{2}(t)}{d t}=-2 i\left[J_{A}(t) \phi_{1}(t)+J_{2} \phi_{3}(t)\right]
$$

So, assuming that at time $t \phi_{j}(t)=\psi_{j}(t)$ for $j=$ $2, \ldots, N-1$, then $\frac{d \phi_{2}(t)}{d t}=\frac{d \psi_{2}(t)}{d t}$ by setting

$$
J_{A}(t)=\frac{\psi_{1}(t)}{\phi_{1}(t)}
$$

and $\frac{d \phi_{N-1}(t)}{d t}=\frac{d \psi_{N-1}(t)}{d t}$ by setting

$$
J_{B}(t)=\frac{\psi_{N}(t)}{\phi_{N}(t)} .
$$

Thus, the practical task of numerically calculating $J_{A}(t)$ and $J_{B}(t)$ involves simulating the evolution of both the $\phi_{j}$ and $\psi_{j}$ states on the original and modified systems respectively, over the time interval $[0, T]$, and evaluating Eqs. (17) and (18).

The functions $J_{A}(t)$ and $J_{B}(t)$ must of course be realvalued, for the Hamiltonian to be Hermitian. Equations (17) and (18) will indeed be real for the $X Y$ chain. The expressions for the $\frac{d \phi_{j}}{d t}$ are all given by a purely imaginary linear combination of the nearest-neighbour values $\phi_{j-1}$ and $\phi_{j+1}$. Then, considering the initial conditions, $\phi_{j}(0)=\delta_{1, j}$, it's clear that the $\phi_{j}(t)$ are real for odd $j$ and imaginary for even $j$, for all values of $t$. The values $\psi_{j}(t)$ also have this property of alternating real and imaginary values. Again, the time derivatives of $\psi_{j}(t)$ are purely imaginary linear combinations of the values $\psi_{j-1}(t)$ and $\psi_{j+1}(t)$. Thus, by performing the change of variables

$$
\psi_{j}^{\prime}(t)= \begin{cases}\psi_{j}(t) & \text { if } j \text { is odd, and } \\ i \psi_{j}(t) & \text { if } j \text { is even, }\end{cases}
$$

the differential equations for $\psi_{j}^{\prime}(t)$ will all have real coefficients. So, the entries of the evolution matrix $e^{-i \tilde{H}^{(1)} T}$ must be real, after that change of variables. Thus, so must be the entries of $P_{\mathcal{B} \cap \mathcal{H}^{(1)}} e^{-i \tilde{H}^{(1)} T} P_{\mathcal{A} \cap \mathcal{H}^{(1)}}$. So, $\vec{w}(T)$, which is the right-singular vector of $P_{\mathcal{B} \cap \mathcal{H}^{(1)}} e^{-i \tilde{H}^{(1)} T} P_{\mathcal{A}^{\prime} \mathcal{H}^{(1)}}$, will also have all real coefficients with respect to the changed variables. Changing variables back, the initial encoded state $\psi_{j}(0)=\vec{w}_{j}(T)$ will thus have the property of having real values for odd $j$ and imaginary values for even $j$, and so will $\psi_{j}(t)$ for all $t$. So, Eqs. (17) and (18) will be real-valued as required.

Eqs. (17) and (18) will never be infinite. In fact, $\left|J_{A}(t)\right|$ and $\left|J_{B}(t)\right|$ will be at most 1 . This is a simple consequence of conservation of probability. Since $\psi_{j}(t)=\phi_{j}(t)$ over the bulk of the chain $(j=2, \ldots, N-1)$ and Alice and Bob's sides only interact via the bulk of the chain for both the physical and modified systems, we have that

$$
\begin{gathered}
\sum_{j=1-N_{P}}^{1}\left|\psi_{j}\right|^{2}=\left|\phi_{1}\right|^{2}, \text { and } \\
\sum_{j=N}^{N+N_{P}}\left|\psi_{j}\right|^{2}=\left|\phi_{N}\right|^{2}
\end{gathered}
$$

from which it follows that $\left|\psi_{1}(t)\right| \leq\left|\phi_{1}(t)\right|$ and $\left|\psi_{N}(t)\right| \leq$ $\left|\phi_{N}(t)\right|$. So, $\left|J_{A}(t)\right| \leq 1$ and $\left|J_{B}(t)\right| \leq 1$, if they are 


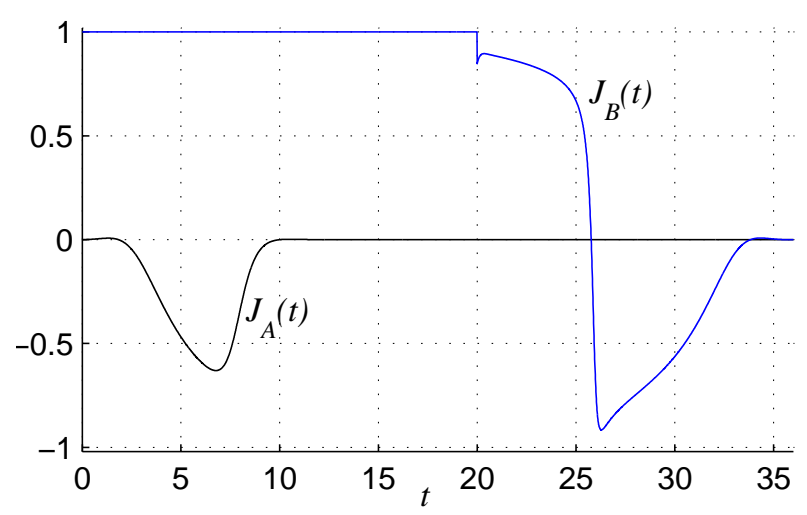

FIG. 6: Control functions for a 104-spin $X Y$ chain, where the non-controlled coupling strengths all have the same strength, 1.

defined. If $J_{A}(t)$ (or $\left.J_{B}(t)\right)$ is undefined $(0 / 0)$, it means that the requirement of $\frac{d \phi_{2}(t)}{d t}=\frac{d \psi_{2}(t)}{d t}$ ( respectively $\left.\frac{d \phi_{N-1}(t)}{d t}=\frac{d \psi_{N-1}(t)}{d t}\right)$ is satisfied regardless of the value of $J_{A}(t)$ (respectively $J_{B}(t)$ ) for that $t$, in which case the value of the control function can be chosen arbitrarily at that time.

We now plot the derived control functions $J_{A}(t)$ and $J_{B}(t)$ for two simple example $X Y$ chain systems. We used numerical integration in these examples, in calculating the evolution of the $\phi_{j}(t)$ due to the time-varying Hamiltonian. We divided the total evolution into a number of discrete time steps, where the approximation was made that the Hamiltonian remains constant throughout each step. The value of $J_{A}(t)$ and $J_{B}(t)$ for a step was calculated from the state of the system at the previous step. We used 2000 time steps, which gave a final fidelity in the physical chain within two significant figures of the correct value given by the evolution of the static modified system.

The first example is a chain 104 spins long (ie. 100 non-controlled spins, plus the four control spins), with all the non-controlled coupling strengths set to the same value, 1 . The control functions were derived by using a modified chain 144 spins long (that is, 20 phantom spins added to each side) with all coupling strengths set to 1 , and the total communication time $T$ chosen to be 36. Figure (6) shows that the resulting $J_{A}(t)$ and $J_{B}(t)$ are quite simple and well behaved. The fidelity measure $\mathcal{C}_{B}(T)$ is 1.0 , to 6 decimal places. This can be compared with the fidelity in the same 104-spin system but without the time-dependent control, that is with $J_{A}(t)$ and $J_{B}(t)$ fixed at 1: over the time interval $0<t<1000$, the value of $\mathcal{C}_{B}(t)$ is at most 0.2809 .

The second example is an $X Y$ chain 29 spins long, but where the non-controlled coupling strengths are randomly sampled uniformly from the interval $[0.95,1.05]$. This is as if the chain has been manufactured with ran-

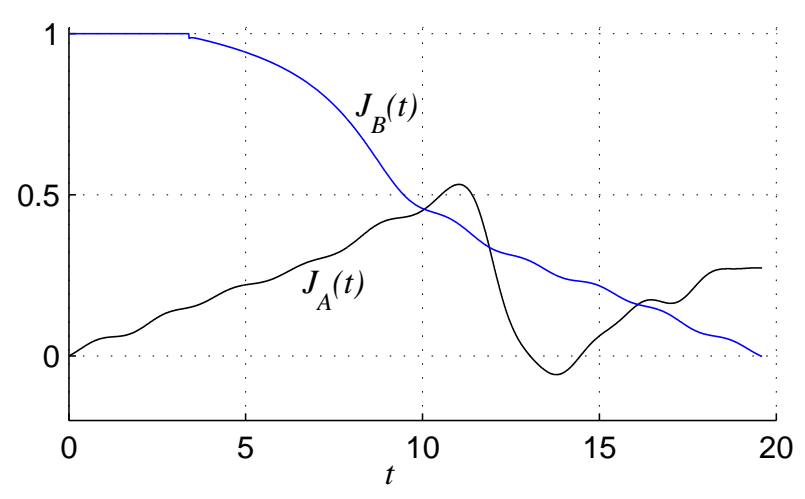

FIG. 7: Control functions for a 29-spin $X Y$ chain, where the non-controlled coupling strengths were chosen randomly from the interval $[0.95,1.05]$.

dom imperfections in the coupling strengths, but these coupling strengths have been somehow measured after the manufacturing process and are known to Alice and Bob. (A shorter chain was chosen in this example, compared with the previous example, in order that a nearperfect fidelity would still result. We have observed that when random couplings are used, the achievable fidelity will decrease as a function of the chain length). We derived control functions using a modified system with 25 phantom spins added to each side, where the new arbitrary coupling strengths are set to 1 . The communication time $T$ was chosen to be 19.5. Figure (7) shows control functions which are a little more complicated in this case, but still rather smooth. The fidelity measure is $\mathcal{C}_{B}(T)=0.99625$. In comparison, in the non-controlled version of this system, with $J_{A}(t)=J_{B}(t)=1$, the value of $\mathcal{C}_{B}(t)$ does not exceed 0.496 over the interval $0<t<1000$. Animations of both examples are available online [12.

What about a system that is not simply an $X Y$ chain, but any configuration conforming to Figure (4) and conserving $Z^{\text {tot }}$ ? Then, the ideas and methods are almost the same, but with the added complication that we need control the $z$ magnetic fields on Alice and Bob's qubits as well as controlling $J_{A}(t)$ and $J_{B}(t)$. The are two reasons why the magnetic control is needed, which we explain for Alice's side. First, the simple phase relation between $\psi_{1}(t)$ and $\phi_{1}(t)$ that we saw in the $X Y$ chain does not occur in general. So, $B_{A 1}(t)$ is chosen simply to keep $\phi_{1}(t)$ in constant relative phase to the $\psi_{1}(t)$. Second, the type of interaction that the $J_{A}(t)$ is modulating may contain it's own magnetic-field-like interactions (that is, nonequal diagonal elements in the Hamiltonian) that need to be cancelled by $B_{A 1}(t)$ and $B_{A 2}(t)$. General expressions for $B_{A 1}(t)$ and $B_{A 2}(t)$ are straightforward to derive, but not particularly illuminating, so will not be given here. 


\section{CONCLUSION}

We have considered the problem of communicating a quantum state over an arbitrary $Z^{\text {tot }}$-conserving spin system. Our first scheme used a static system Hamiltonian, and utilised the fact that the sender and receiver control several spins each, to increase fidelity by performing state encoding. We showed that choosing the optimal state encoding is a simple matter of performing a SVD on a modified evolution matrix.

We have also shown that if the sender and receiver have control of just two spins each, but can vary the interactions on these four spins over time, then they can achieve a fidelity that is equal to if they each controlled many more spins on a static system and used the optimal state encoding. We have given a practical method of deriving suitable control functions. The advantage of this scheme is the "fixed interface" that Alice and Bob have with the chain. That is, if the chain is altered, the only change that Alice and Bob need make is to their control functions, rather than to the number of spins they control.

It should be noted that the systems we have considered are idealised to a high degree. In particular, we haven't considered the effects of external noise, or the effect of having a Hamiltonian that only approximately commutes with $Z^{\text {tot }}$, or the case where Alice and Bob have only an approximate knowledge of the system Hamiltonian. These issues will be the subject of future work by the author.

\section{Acknowledgements}

I would like to thank Tobias Osborne and Michael Nielsen for their detailed comments on the original manuscript, and for helpful and enlightening discussions relating to this work.

\section{APPENDIX A}

Here we outline a proof of the claim in Sec. III regarding the connection between $\mathcal{C}_{B}(T)$ and the system's ability to transmit entanglement from Alice to Bob. This connection helps establish $\mathcal{C}_{B}(T)$ as a good measure of communication fidelity.

Proposition. Suppose that Alice sends a state which is maximally entangled with some additional spin that Alice possesses. (After the maximally entangled state is created, the additional spin is assumed to not interact during the remainder of the communication procedure). Then, after the communication procedure of Sec. [I] is carried out, the entanglement (measured by concurrence) between Alice's additional spin and Bob's decoded message, equals $\sqrt{\mathcal{C}_{B}(T)}$.

Proof: Note that it doesn't matter which maximallyentangled state is used - all such states are equivalent up to a local unitary on the additional spin, and such a local unitary could not possibly affect the way entanglement is transferred through the system.

Let the additional spin "+" and the spin " $M$ " containing the message have the maximally entangled state $\frac{1}{\sqrt{2}}\left(|0\rangle_{+}|0\rangle_{M}+|1\rangle_{+}|1\rangle_{M}\right)$. Thus, after Alice performs her encoding, the entire state is:

$$
|\Phi(0)\rangle=\frac{1}{\sqrt{2}}\left[|0\rangle_{+}|\mathbf{0}\rangle_{A}|\mathbf{0}\rangle_{\bar{A}}+|1\rangle_{+}\left|1_{E N C}\right\rangle_{A}|\mathbf{0}\rangle_{\bar{A}}\right] .
$$

After the system evolves for time $T$, the state becomes

$$
\begin{aligned}
|\Phi(T)\rangle= & \frac{1}{\sqrt{2}}\left[|0\rangle_{+}|\mathbf{0}\rangle_{\bar{B}}|\mathbf{0}\rangle_{B}+|1\rangle_{+}\left(\sqrt{1-\mathcal{C}_{B}(T)}|\eta(T)\rangle\right.\right. \\
& \left.\left.+\sqrt{\mathcal{C}_{B}(T)}|\mathbf{0}\rangle_{\bar{B}}|\gamma(T)\rangle_{B}\right)\right] .
\end{aligned}
$$

Then Bob performs a decoding unitary, denoted $U_{\mathrm{dec}}$, on the spins he controls. $U_{\mathrm{dec}}$ is defined to act as follows: $U_{\mathrm{dec}}|\mathbf{0}\rangle_{B}=|\mathbf{0}\rangle_{B}$ and $U_{\mathrm{dec}}|\gamma(T)\rangle_{B}=|0 \ldots 01\rangle_{B}$, where $|0 \ldots 01\rangle_{B}$ is the $|1\rangle$ state on spin $N$ and the all-zero state on Bob's other spins. After Bob's decoding, the joint state of Alice's additional spin and Bob's decoded spin is:

$$
\begin{aligned}
& \rho_{+/ N}=\operatorname{tr}_{\overline{+/ N}}\left(U_{\operatorname{dec}} \operatorname{tr}_{\bar{B}}(|\Phi(T)\rangle\langle\Phi(T)|) U_{\mathrm{dec}}^{\dagger}\right) \\
& =\frac{1}{2}\left[\left(1-\mathcal{C}_{B}(T)\right)|1\rangle\left\langle 1\left|\otimes \tilde{\rho}+\mathcal{C}_{B}(T)\right| 11\right\rangle\langle 11|\right. \\
& +|00\rangle\langle 00|+\sqrt{\mathcal{C}_{B}(T)}(|00\rangle\langle 11| \\
& +|11\rangle\langle 00|)] \text {, }
\end{aligned}
$$

where $\tilde{\rho} \equiv \operatorname{tr} \overline{+/ N}\left(U_{\operatorname{dec}} \operatorname{tr}_{\bar{B}}(|\eta(T)\rangle\langle\eta(T)|) U_{\mathrm{dec}}^{\dagger}\right)$, and where $\operatorname{tr}_{(\cdot)}(\cdot)$ is the partial trace performed over the spins indicated.

Concurrence is a measure of entanglement between two qubits [13]. The value of concurrence for a density matrix $\rho_{+/ N}$ is equal to

$$
E\left(\rho_{+/ N}\right)=\max \left\{0, \sqrt{\lambda_{1}}-\sqrt{\lambda_{2}}-\sqrt{\lambda_{3}}-\sqrt{\lambda_{4}}\right\},
$$

where the $\lambda_{j}$ s are the eigenvalues, in nonincreasing order, of the matrix $\rho_{+/ N}\left(\sigma^{y} \otimes \sigma^{y}\right) \rho_{+/ N}^{*}\left(\sigma^{y} \otimes \sigma^{y}\right)$, where * represents complex conjugation in the computational basis. It can be shown that

$$
\begin{aligned}
& \lambda_{1}=\frac{1}{4}\left(\sqrt{\tilde{\rho}_{11} \mathcal{C}_{B}(T)+1-\tilde{\rho}_{11}}+\sqrt{\mathcal{C}_{B}(T)}\right)^{2} \\
& \lambda_{2}=\frac{1}{4}\left(\sqrt{\tilde{\rho}_{11} \mathcal{C}_{B}(T)+1-\tilde{\rho}_{11}}-\sqrt{\mathcal{C}_{B}(T)}\right)^{2} \\
& \lambda_{3}=0 \\
& \lambda_{4}=0,
\end{aligned}
$$

where $\tilde{\rho}_{11}=\langle 0|\tilde{\rho}| 0\rangle$. Thus, using the fact that $\mathcal{C}_{B}(T)$ and $\tilde{\rho}_{11}$ each lie in the interval $[0,1]$, we have

$$
E\left(\rho_{+/ N}\right)=\sqrt{\mathcal{C}_{B}(T)},
$$

as required. 
[1] D. P. DiVincenzo, Fortschritte der physik - progress of physics 48, 771 (2000), arXiv quant-ph/0002077

[2] J. Preskill, Physics 229: Advanced mathematical methods of physics - Quantum computation and information (California Institute of Technology, Pasadena, CA, 1998), http://www.theory.caltech.edu/people/preskill/ph229/.

[3] M. A. Nielsen and I. L. Chuang, Quantum computation and quantum information (Cambridge University Press, Cambridge, 2000).

[4] S. Bose, Phys. Rev. Lett. 91, 207901 (2003), arXiv quant-ph/0212041.

[5] M. Christandl, N. Datta, A. Ekert, and A. J. Landahl, arXiv quant-ph/0309131 (2003).

[6] T. J. Osborne and N. Linden, arXiv quant-ph/0312141 (2003).
[7] T. J. Osborne and S. Severini, arXiv:quant-ph/043127 (2004).

[8] R. A. Horn and C. R. Johnson, Topics in matrix analysis (Cambridge University Press, Cambridge, 1991).

[9] J. Kempe, Contemp. Physics 44, 307 (2003).

[10] A. Ambainis, arXiv quant-ph/0311001 (2003).

[11] A. M. Childs, R. Cleve, E. Deotto, E. Farhi, S. Gutmann, and D. A. Spielman, in Proc. $35^{\text {th }}$ Annual ACM Symposium on Theory of Computing (ACM, New York, 2003), pp. $59-68$.

[12] H. L. Haselgrove (2004), online animations available at http://www.physics.uq.edu.au/people/hlh/comms

[13] W. K. Wootters, Phys. Rev. Lett. 80, 2245 (1998). 